\title{
SISTEMA DE MONITORAMENTO DE TEMPERATURA CORPORAL PARA ATIVIDADES COM EXPOSIÇÃO AO FRIO ARTIFICIAL CONTROLADO
}

\section{BODY TEMPERATURE MONITORING SYSTEM FOR ACTIVITIES WITH CONTROLLED ARTIFICIAL COLD EXPOSURE}

\author{
Fabiano Takeda* E-mail: takeda.f@bol.com.br \\ Antônio Renato Pereira Moro* E-mail: renato.moro@ufsc.br \\ Saulo Guths* E-mail: saulo.guths@Impt.ufsc.br \\ *Universidade Federal de Santa Catarina (UFSC), Florianópolis, SC
}

\begin{abstract}
Resumo: O objetivo desse estudo foi utilizar um sistema completo para avaliar as condições ambientais e o desconforto térmico dos trabalhadores com exposição a baixas temperaturas artificiais. O sistema proposto consiste em uma rede de sensores sem fio (WSN) usando a tecnologia Zigbee, fornecendo um conjunto de sensores para medir simultaneamente a temperatura corporal em diferentes regiões do corpo de trabalhadores expostos a baixas temperaturas e para medir as condições ambientais que influenciam na avaliação de desconforto térmico. Os dados foram coletados por um módulo USB Zeegbee conectado a um PC com Windows e os resultados são gravados e exibidos em tempo real. O estudo foi realizado com avaliações em 153 trabalhadores de linha de corte de um frigorifico de abate e cortes de frangos. O sistema apresentado foi comprovado como uma maneira fácil e rápida para instalar e confiável para avaliar a exposição ao frio artificial. Os resultados mostraram que apesar da atividade representar um PMV adequado, atendimento aos critérios legais e normativos nacionais, os resultados do dedo da mão e do hálux apresentam condições de frio e dor durante quase toda jornada de trabalho.
\end{abstract}

Palavras-chave: Frio. Monitoramento de temperatura. ISO 7730/05.

Abstract: The objective of this study was to use a complete system to evaluate the environmental conditions and the thermal discomfort of workers with exposure to low artificial temperatures. The proposed system consists of a wireless sensor network (WSN) using Zigbee technology, providing a set of sensors to simultaneously measure body temperature in different regions of the body of workers exposed to low temperatures and to measure the environmental conditions that influence the evaluation of thermal discomfort. The data was collected by a Zeegbee USB module connected to a Windows PC and the results are recorded and displayed in real time. The study was carried out with evaluations in 153 workers of cut line of a refrigerator of slaughter and cuts of chickens. The presented system has been proven as an easy and quick to install and reliable way to assess artificial cold exposure. The results showed that although the activity represents an adequate PMV, in compliance with national legal and normative criteria, the results of the finger and the hallux show conditions of cold and pain during almost every working day.

Keywords: Cold. Temperature monitoring. ISO 7730/05.

\section{INTRODUÇÃO}

A indústria de abate e processamento de carnes faz atualmente do Brasil um dos principais exportadores mundiais de produtos de origem animal. O setor cresce continuamente em volume de produção, empresas e número de funcionários e 
contribui significativamente para o produto interno bruto em muitos países (IBGE, 2017; OLIVEIRA; MENDES, 2014). Este aumento está vinculado à demanda por proteína animal principalmente da carne de frango devido ao rótulo saudável e acessível, devido à demanda do mercado de carnes, inclusive internacional, que tem pressionado a chamada competitividade (HARMSE; ENGELBRECHT; BEKKER, 2016). A maioria da carne de frango exportada brasileira é sob a forma de cortes $(54,7 \%)$ e o restante consiste em carne processada (4,6\%), carne temperada $(4,5 \%)$ e frango inteiro $(36,2 \%)$ (BRAZILIAN POULTY ASSOCIATION, 2015).

No Brasil, a avicultura emprega mais de 3,6 milhões de pessoas, principalmente trabalhadores de linha de produção. Devido à falta de máquinas que fornecem automação total no setor, a maioria da carne de frango produzida no Brasil é processada através de mão-de-obra manual, usando ferramentas manuais, como facas com pontas vivas e tesouras de corte (PIEDRAHITA, 2003).

Nesta perspectiva, a maior incidência de acidentes e lesões relacionadas às doenças de trabalhadores em frigoríficos e abatedouros está direcionada nos membros superiores, consequência do modelo de atividade exercida pelos trabalhadores (ROSSI, 2008). Além disso, nos frigoríficos, em diversas atividades, as mãos dos trabalhadores sofrem uma grande influência do frio devido ao contato direto com as peças de frangos que estão em temperaturas que dependendo do setor e da atividade podem estar abaixo de $10^{\circ} \mathrm{C}$.

Com relação ao agente ambiental frio, em geral, as normativas de saúde e segurança do trabalho estipulam parâmetros e dados quantitativos para controle somente do ambiente de trabalho e especificamente a Portaria 210 de 10/11/1998 do Ministério da Agricultura e do Abastecimento do Brasil, obrigatória para atividades de cortes de carnes, também recomenda valores de temperatura no ambiente de trabalho no qual uma sala de cortes de frangos a temperatura do frango não pode ser superior à $7^{\circ} \mathrm{C}$, e a temperatura ambiente não pode ser superior aos $12^{\circ} \mathrm{C}$ (Portaria $\mathrm{n}^{\circ} 210 \mathrm{de}$ 10/11/1998).

Cabe ressaltar que nas atividades executas em abatedouros e frigoríficos, o desconforto causado pelo frio pode, em alguns casos, ser contornado pelo próprio indivíduo, que sobrepõe de vestimenta até sentir-se confortável, por exemplo, o uso de blusas e calças embaixo dos uniformes cedidos pelas empresas, porém, isto não acontece com as extremidades, principalmente com as mãos que necessitam de 
agilidade para exercer suas atividades de rotina, pois a sobreposição de luvas dificulta os movimentos necessários de motricidade fina dos dedos e mãos (HOLMÉR, 1997).

A exposição dos trabalhadores a ambientes considerados frios nas atividades executadas em frigoríficos e abatedouros promove perdas de calor no corpo devido à exposição ao frio ser maior que o ganho de temperatura, isso ocorre em diversos postos de trabalho principalmente com atividade estática que não contribui na geração de calor, acarretando em riscos à saúde do trabalhador (HOLMÉR, 1997).

Além dos riscos à saúde, uma das primeiras consequências da exposição ao frio abaixo dos limites de tolerância é o desconforto seguido de dor na região atingida (ISO 13732-3, 2005). Esses sintomas podem se agravar, de acordo com o tempo de exposição ou dependendo de quão baixa seja a temperatura, e se não forem tomadas ações de controle pode ocorrer o aparecimento de doenças. Diversas são as doenças citadas na literatura médica que podem ser causadas devido à exposição de trabalhadores ao frio durante a jornada de trabalho em ambientes sem as condições térmicas adequadas. As doenças comumente citadas na literatura médica relacionadas com a exposição ao frio são: Ulcerações, Frostbite, Fenômeno de Raynaud, Urticária pelo Frio, Frieiras (Perniose), Doenças Respiratórias, entre outras (SALIM, 2003).

Dentro desse contexto, o objetivo desse estudo foi utilizar um sistema completo para avaliar as condições ambientais e o desconforto térmico dos trabalhadores com exposição a baixas temperaturas artificiais.

\section{MATERIAIS E METÓDOS}

Buscou-se com esta pesquisa avaliar a exposição ao frio ocupacional utilizando um sistema e equipamentos de monitoramento de temperatura ambiental e corporal, que foi desenvolvido, construído, calibrado e testado por uma equipe técnica do laboratório de meios porosos e propriedades termofísicas (LMPT) da Universidade Federal de Santa Catariana (GÜTHS et al., 2017).

Os equipamentos e o sistema proposto consistem em uma rede de sensores sem fio (WSN) usando a tecnilogia Zigbee, no qual um conjunto de sensores se destina a medir a temperatura corporal dos trabalhadores, enquanto o outro conjunto avalia as variáveis ambientais. 
Ambos os módulos de sensores enviam os dados amostrados em tempo real através de um link de rádio através de um conversor USB-serial conectado a um computador.

No estudo foram utilizados dois módulos de sensores em cada trabalhador avaliado (item 3 - Quadro 01), o qual um módulo foi utilizado para medir a temperatura no dedo indicador, centro da mão, punho e ouvido e o outro módulo foi utilizado para medir a temperatura do hálux e região central na parte superior do pé. Em cada dia de avaliação foram monitorados dois trabalhadores durante toda a jornada diária.

Os termistores foram fixados com fita microporosa diretamente na pele do trabalhador (item 2 - Quadro 01). O termistor de temperatura da orelha foi inserido em um fone de ouvido de silicone (item 1 - Quadro 01).

Após a instalação dos termistores, os trabalhadores foram vestidos pelos pesquisadores dos demais equipamentos de proteção obrigatórios para executar suas atividades diárias, a ilustração de um trabalhador com os módulos de sensores e todos os equipamentos para executar suas atividades é representado no item 3 - Quadro 01.

O módulo de medição das variáveis ambientais foi montado próximo às estações de trabalho onde foram coletados os dados de temperatura corporal, conforme ilustrado na figura apresentada no item 4 - Quadro 01. 
Quadro 1 - Demonstração dos equipamentos de monitoramento e temperatura instalados nos trabalhadores e nos postos de trabalho

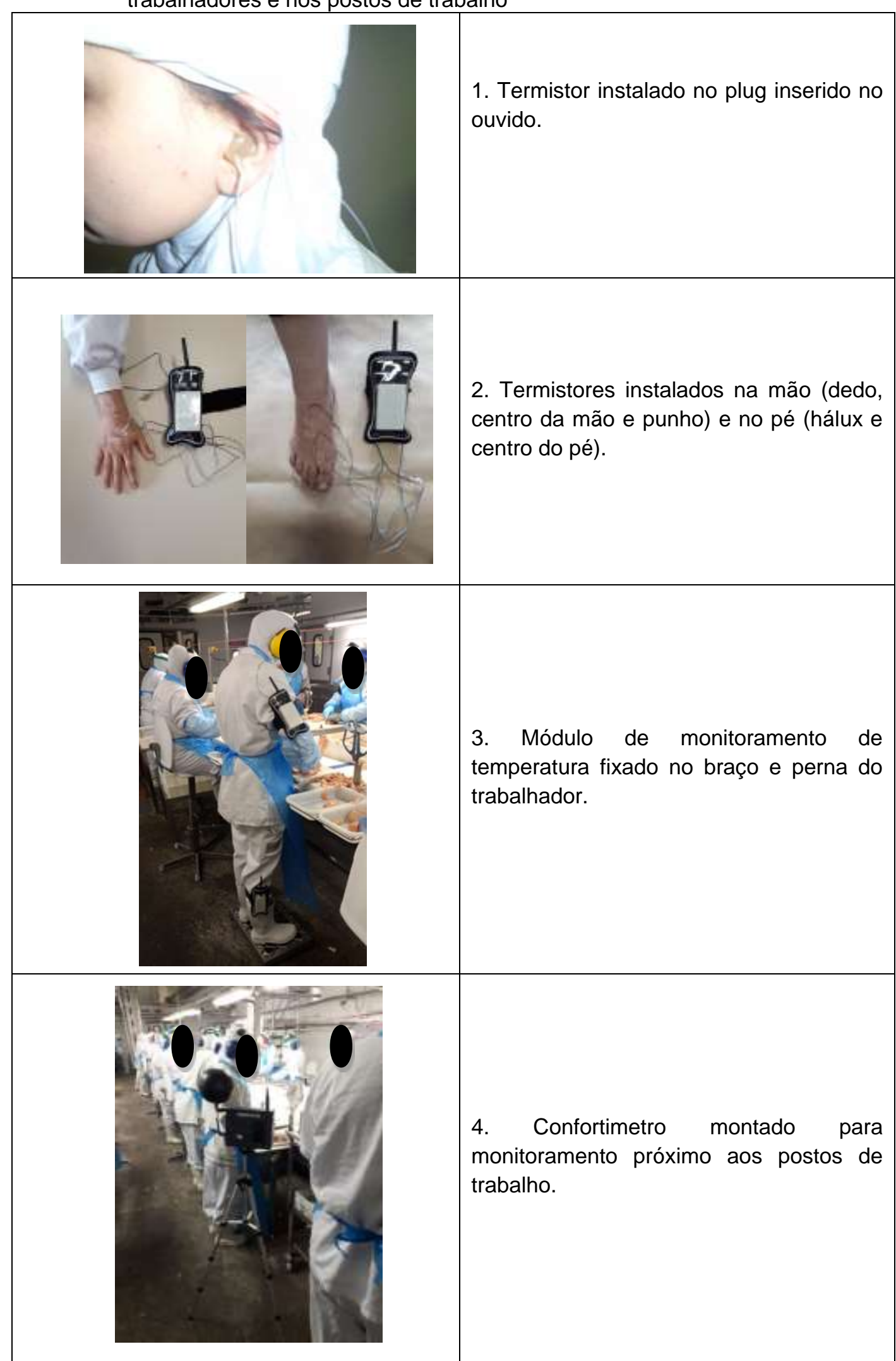

Fonte: Dados da pesquisa (2018) 
As variáveis ambientais coletadas pelo módulo de medição ambiental determinam dados que subsidiam a avaliação de PMV (Voto Médio Estimado) e PPD (Voto Médio Predito). Esses índices são definidos pela ISO 7730 (2005) e ASHRAE 55 (2013), com base em estudos de Fanger (1970), para avaliação de conforto térmico de uma pessoa ou um grupo de pessoas que praticam uma atividade.

A amostra foi realizada com 153 trabalhadores, no qual todos participantes fazem parte do quadro do setor de cortes, das linhas de produção de peito, perna e asa. Não foram avaliados trabalhadores com menos de um mês de empresa ou em desempenho das atividades. A pesquisa foi realizada em um frigorífico de abate e processamento de frangos localizado na região de Santa Catarina.

A jornada de trabalho nas linhas de produção durante o período de coleta de dados iniciava-se às 06h: 00min, com a primeira pausa ocorrendo no intervalo entre 07h: $20 \mathrm{~min}$ às $07 \mathrm{~h}$ : $40 \mathrm{~min}$, segunda pausa entre $09 \mathrm{~h}: 20 \mathrm{~min}$ e $09 \mathrm{~h}$ : $40 \mathrm{~min}$, intervalo de refeição entre $11 \mathrm{~h}$ : $20 \mathrm{~min}$ às $12 \mathrm{~h}: 20 \mathrm{~min}$, a terceira pausa entre $14 \mathrm{~h}: 00 \mathrm{~min}$ e $14 \mathrm{~h}$ : $20 \mathrm{~min}$ e término da jornada às $15 \mathrm{~h}: 48 \mathrm{~min}$. A jornada é de $8 \mathrm{~h}$ : $48 \mathrm{~min} / \mathrm{dia}$, com intervalo de 60 minutos para alimentação e com pausas de recuperação psicofisiológica de 60 minutos distribuídas na jornada de trabalho em três pausas de 20 minutos, para cumprimento da NR 36 - Segurança e Saúde no Trabalho em Empresas de Abate e Processamento de Carnes e Derivados (NR 36, 2014).

Para realização do estudo foi disponibilizado uma sala com temperatura ambiente média de $23,2^{\circ} \mathrm{C}$, esta temperatura foi medida com um medidor de estresse térmico marca Instrutherm, modelo TGD-300.

Cada trabalhador participante da pesquisa antes de iniciar suas atividades na linha de produção foi direcionado para a sala onde foram instalados os sensores. Ao chegar à sala o procedimento realizado pelos pesquisadores consistia em retirar a botina e a meia do pé do trabalhador, instar os sensores, recolocar a meia e botina e em seguida instalar os sensores na mão e ouvido. O tempo médio de instalação de todos os sensores foi de aproximadamente 5 minutos por trabalhador, instalados por dois pesquisadores. Durante toda a jornada, intervalo e pausas o trabalhador permaneceu com os sensores instalados e somente no final da jornada retornava para a sala para retirada dos sensores e para o preenchimento dos dados do perfil do trabalhador. 
Durante todo o tempo de trabalho na linha de produção, as atividades desenvolvidas pelos trabalhadores participantes do estudo consistiram em pegar e manipular as peças de frango com uma das mãos, de forma que facilite o direcionamento do corte com a faca sustentada com a mão contrária.

Os sensores foram instalados em todos trabalhadores na mão que segura 0 produto, no pé e ouvido correspondentes ao mesmo lado (direito ou esquerdo) dos sensores da mão.

Os pontos corporais avaliados na mão e pé foram selecionados fundamentados na ISO 11079 (2007) "Ergonomics of the termal environment" a qual não determina pontos específicos a serem avaliados para exposições ao frio localizado, mas recomenda controle frequente de temperatura dos dedos e sugere que as temperaturas devem estar acima de $24^{\circ} \mathrm{C}$ para a preservação e bom funcionamento das mãos. Também determina que a quantidade de conhecimento sobre as respostas a resfriamento local é insuficiente para o desenvolvimento de um método de avaliação único e que pesquisas sobre o assunto devem ser incentivadas (ISO 11079, 2007).

Para mensuração da temperatura superficial do produto manipulado foi utilizado um termômetro digital infravermelho marca Minipa, modelo MT-350.

Conforme análise de risco descrita no programa de prevenção de riscos ambientais (PPRA) da empresa, em todas as atividades avaliadas nessa pesquisa é obrigatória a utilização na mão que segura o produto: luva de algodão sobreposta com luva nitrílica sobreposta com luva malha de aço e na mão que segura à faca, luva de algodão sobreposta com luva nitrílica. Nos pés, meia de algodão e botina de Policloreto de Vinila (PVC), todos os equipamentos são fornecidos pela empresa. A meia possui certificação para exposição ao frio e a botina possui certificação para exposição à umidade. Durante a pesquisa foram entregues meias e luvas para os trabalhadores que por algum motivo específico não possuíam a luva ou meia conforme determinação do PPRA. Todos foram entregues apenas no dia da avaliação no trabalhador e para os que utilizam meias e luvas adicionais foram solicitados que não utilizassem no dia da avaliação apenas.

Para compilação e análise dos dados de temperatura dos trabalhadores foram separados os resultados de cada subsetor: produção de peito, perna e asa. Utilizouse da estatística descritiva com calculo de média, desvio padrão e exclusão dos outliers (critério $|\mathrm{Z}|>3$ = Valor outliers). 
Ressalta-se que nesse estudo não foi realizada análise separada dos dados outliers para investigar os casos extremos.

Para comparação das médias dos resultados das avaliações dos trabalhadores de cada subsetor foi utilizado o resultado conforme atendimento do calculado do teste F na análise de variância (ANOVA) com nível de significância de 5\%.

Foram utilizados os valores médios calculados dos resultados das avaliações no início da atividade, saída e retorno de cada pausa psicofisiológica, saída e retorno do intervalo da refeição e término da jornada de trabalho.

Como parâmetro para análise dos resultados das avaliações de temperatura em cada sítio corporal foi utilizada uma adaptação das escalas de temperatura, baseada no estudo conduzido por Lehmuskallio, Hassi e Kettunen (2002) onde a faixa de temperatura entre $36-34^{\circ} \mathrm{C}$ indica condição de conforto térmico, entre 33,9-29ำ indica condição neutra, entre 28,9 - 25ํㅡ indica desconforto (resfriado), entre 24,9 $18^{\circ} \mathrm{C}$ indica sensação de desconforto (frio), entre $17,9-12^{\circ} \mathrm{C}$ indica sensação de dor provocada pelo frio, entre 11,9 - $8^{\circ} \mathrm{C}$ indica sensação de dor e formigamento (perda gradual da percepção tátil) e menor de 8ㄷ indica sensação de dor e congelamento.

A pesquisa foi autorizada pelo representante legal da empresa que concedeu o local para realizar as avaliações e todas as funcionárias participantes aceitaram o termo de consentimento livre esclarecido conforme protocolo da pesquisa, submetida e aprovada pela Comissão de Ética em Pesquisa da Universidade Federal de Santa Catarina, atendendo a todas as recomendações legais. $O$ anonimato $e$ confidencialidade das informações foram mantidos nos registros dos dados.

\section{RESULTADOS}

Inicialmente são apresentados na Tabela 01 os resultados referentes ao perfil dos trabalhadores. 
Tabela 1 - Resultados do perfil dos trabalhadores participantes do estudo

\begin{tabular}{|c|c|c|}
\hline \multirow{2}{*}{$\frac{\text { Características }}{\text { Sexo }}$} & \multicolumn{2}{|c|}{ Dados } \\
\hline & (n) & $(\%)$ \\
\hline Masculino & 62 & 40,5 \\
\hline Feminino & 91 & 59,5 \\
\hline \multicolumn{3}{|l|}{ Idade } \\
\hline Até 20 anos & 8 & 5,2 \\
\hline Acima de 20 até 30 anos & 44 & 28,8 \\
\hline Acima de 30 até 40 anos & 53 & 34,7 \\
\hline Acima de 40 até 50 anos & 36 & 23,5 \\
\hline Acima de 50 anos & 12 & 7,8 \\
\hline \multicolumn{3}{|c|}{ Tempo de empresa (meses) } \\
\hline Até 12 & 40 & 26,1 \\
\hline 12 a 36 & 28 & 18,3 \\
\hline 36 a 60 & 29 & 19,0 \\
\hline 60 a 120 & 22 & 14,4 \\
\hline$>120$ & 34 & 22,2 \\
\hline \multicolumn{3}{|l|}{ Grau de instrução } \\
\hline Analfabeto & 0 & 0,0 \\
\hline Ensino fundamental incompleto & 19 & 13,8 \\
\hline Ensino fundamental completo & 34 & 24,6 \\
\hline Ensino médio incompleto & 29 & 21,0 \\
\hline Ensino médio completo & 38 & 27,5 \\
\hline Superior incompleto & 13 & 9,4 \\
\hline Superior completo & 5 & 3,6 \\
\hline
\end{tabular}

Nomenclaturas: $\mathrm{n}$ - número.

Fonte: Dados da pesquisa (2018)

Em síntese os resultados apontam que há mais trabalhadores do sexo feminino no setor, que a idade que prevalece é acima de 30 até 40 anos, com relação ao tempo de empresa percebe-se nos resultados que é bem distribuído conforme os parâmetros estabelecidos no estudo, o grau de ensino também apresenta resultados bem distribuídos entre ensino fundamental incompleto até ensino médio completo. Percebe-se nos resultados que é baixo o percentual de trabalhadores com grau de instrução superior, provavelmente devido à demanda de capacitação exigida para o cargo.

Em seguida são apresentados os resultados calculados dos dados do ambiente de trabalho (temperatura, velocidade do ar e umidade relativa), temperatura do produto manuseado, sendo que apenas esse último não foi mensurado com os equipamentos desenvolvidos no LMPT. Ressalta-se que os resultados desses fatores 
podem influenciar diretamente nas temperaturas medidas nos sítios corporais dos trabalhadores durante a jornada de trabalho.

Gráfico 1 - Resultados do monitoramento ambiental

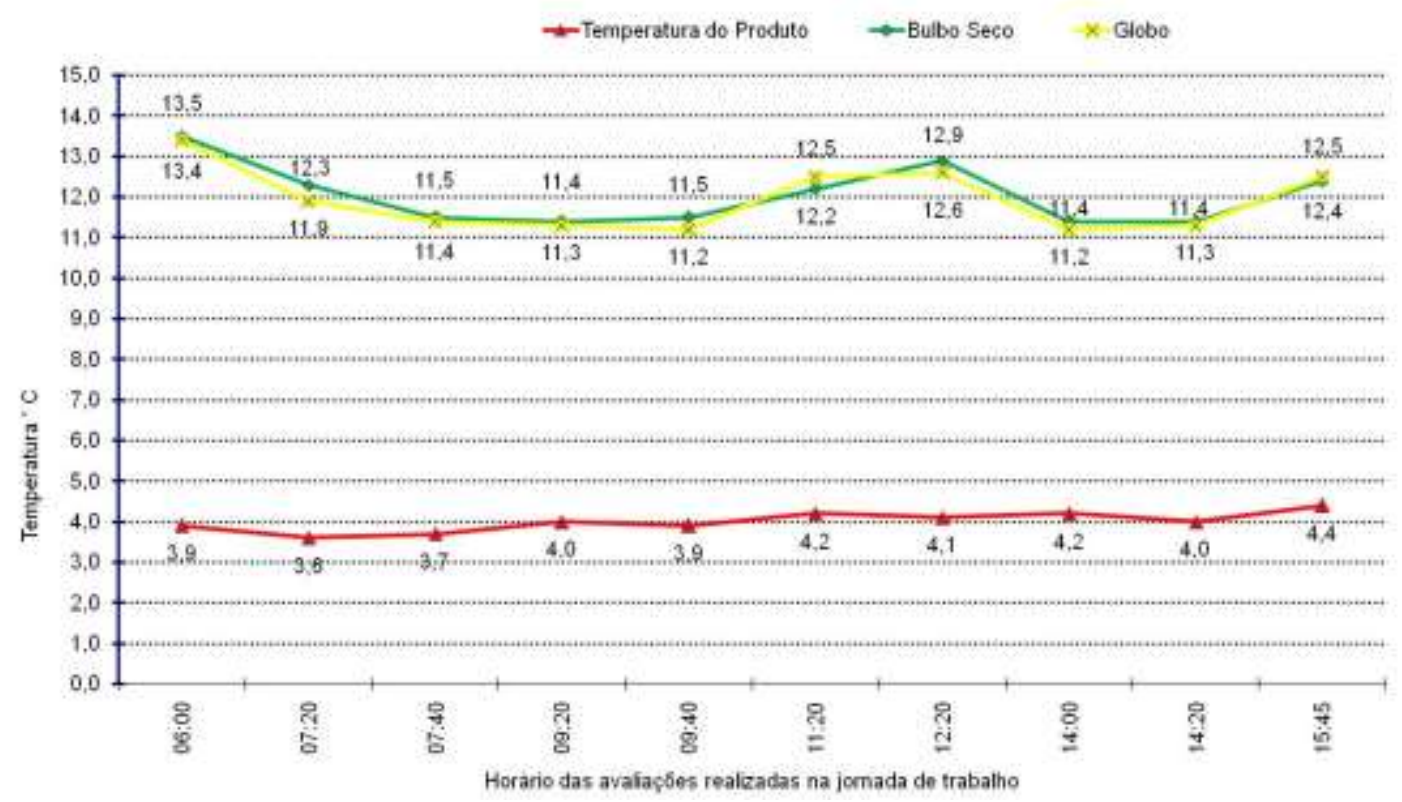

Fonte: Dados da pesquisa (2018)

Verifica-se que conforme resultados das avaliações apresentados no Gráfico 1, a média da temperatura do produto apresenta a menor temperatura de 3,7ํㅡ após o retorno da primeira pausa psicofisiológica e a maior média de temperatura no final da jornada de trabalho com valor médio de $4,4^{\circ} \mathrm{C}$.

As médias de temperaturas de bulbo seco registraram os menores resultados com $11,4^{\circ} \mathrm{C}$ na saída da segunda e retorno da terceira pausa psicofisiológica e as maiores médias das temperaturas ocorreram no início da jornada de trabalho com resultado de $13,5^{\circ} \mathrm{C}$.

As temperaturas de termômetro de globo registraram os menores resultados com média de $11,2^{\circ} \mathrm{C}$ no retorno da segunda e saída da terceira pausa psicofisiológica. As maiores médias das temperaturas ocorreram no início da jornada de trabalho com $13,4^{\circ} \mathrm{C}$.

A velocidade do ar e a umidade relativa do ar não apresentaram resultados com variações significativas durante toda jornada de trabalho, condição que confirma o eficiente controle de temperatura no ambiente de trabalho avaliado. A velocidade do ar apresentou à menor média com $0,3 \mathrm{~m} / \mathrm{s}$ às $12 \mathrm{~h}$ : $20 \mathrm{~min}$ e a maior média com $0,36 \mathrm{~m} / \mathrm{s}$ 
em três momentos das avaliações conforme critérios estabelecidos nesse estudo. Já a umidade relativa apresentou a menor média com $82 \%$ e a maior média com $85 \%$.

As temperaturas mensuradas nos sítios corporais localizados nas mãos estão apresentadas no Gráfico 2.

Gráfico 2 - Resultados do monitoramento de temperatura no dedo indicador, centro da mão, punho e ouvido.

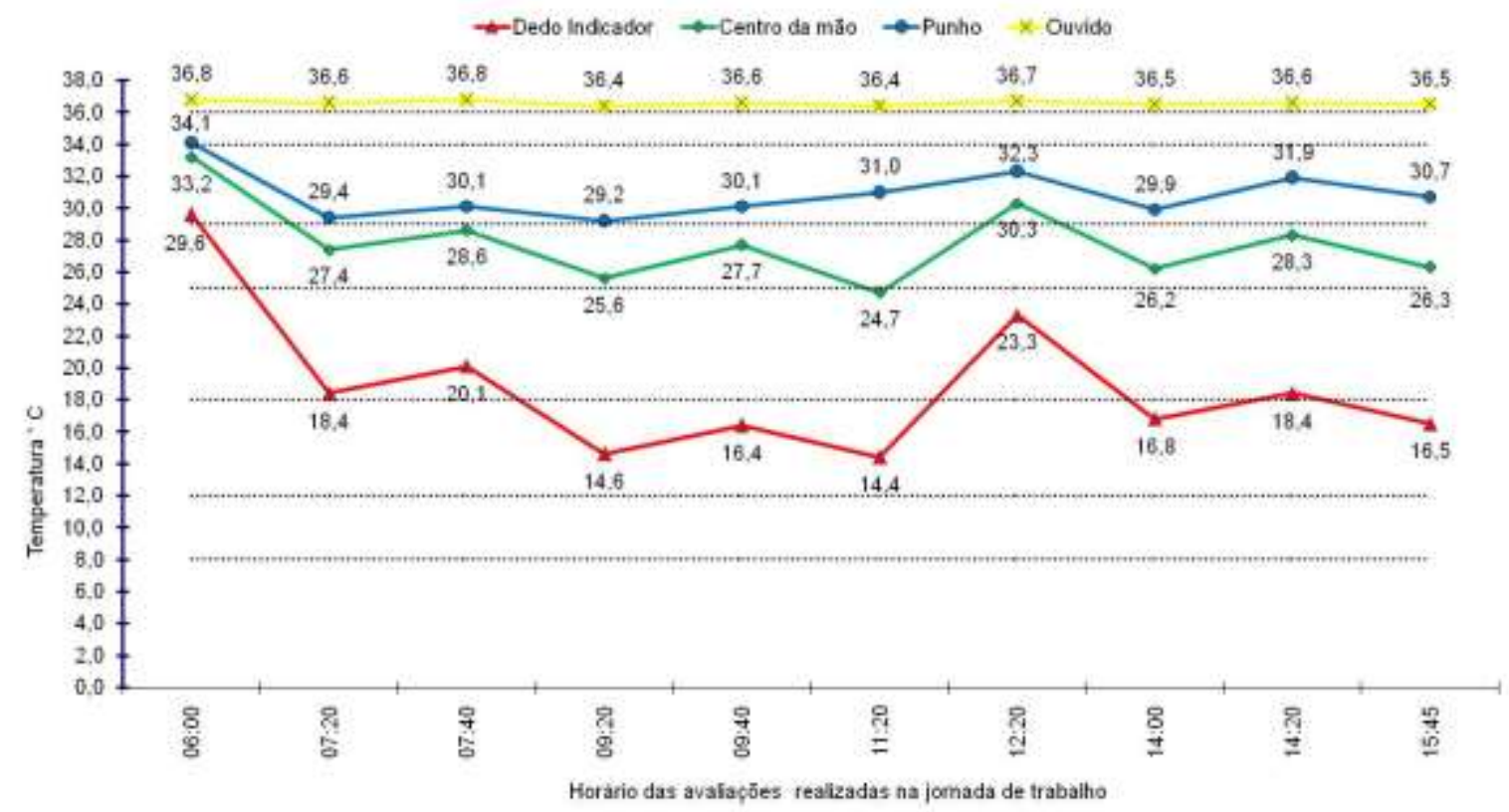

Fonte: Dados da pesquisa (2018)

Os resultados das médias das avaliações no dedo indicador apresentam as menores temperaturas com $14,4^{\circ} \mathrm{C}$ na saída para pausa para refeição e as maiores temperaturas com $29,6^{\circ} \mathrm{C}$ no início da jornada de trabalho.

Os resultados das médias das avaliações no centro da mão apresentam as menores temperaturas com $24,7^{\circ} \mathrm{C}$ na saída para pausa para refeição e as maiores temperaturas com $33,2^{\circ} \mathrm{C}$ no início da jornada de trabalho.

Os resultados das médias das avaliações no punho apresentam as menores temperaturas com $29,2^{\circ} \mathrm{C}$ na saída para segunda pausa psicofisiológica e as maiores

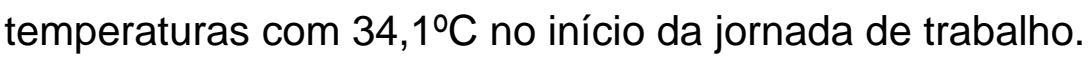

Os resultados das médias das avaliações no ouvido não apresentam variação significativa durante toda jornada de trabalho.

No Gráfico 3 é apresentado os resultados das médias das avaliações realizadas nos sítios corporais do pé de cada trabalhador. 
Gráfico 3 - Resultados do monitoramento de temperatura no hálux, centro do pé e ouvido.

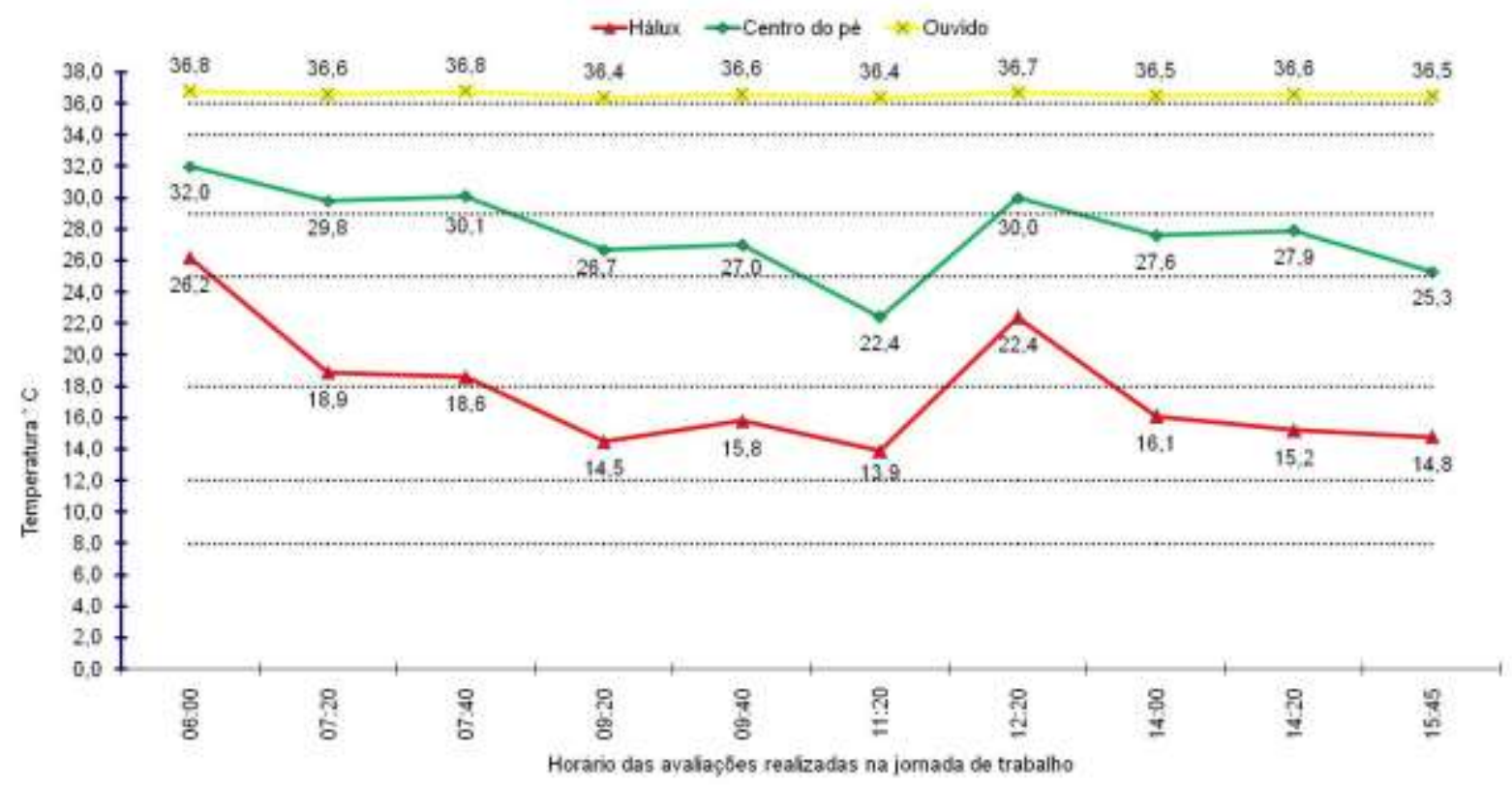

Fonte: Dados da pesquisa (2018)

Os resultados das médias das avaliações no hálux apresentam as menores temperaturas com $13,9^{\circ} \mathrm{C}$ na saída da pausa para refeição e as maiores temperaturas com $26,2^{\circ} \mathrm{C}$ no início da jornada de trabalho.

Os resultados das médias das avaliações no centro do pé apresentam as menores temperaturas com $22,4^{\circ} \mathrm{C}$ na saída da pausa para refeição e as maiores temperaturas com $32,0^{\circ} \mathrm{C}$ no início da jornada de trabalho.

\section{DISCUSSÃo}

Inicialmente percebe-se que considerando os requisitos legais das leis e normas nacionais de saúde e segurança do trabalho, ao analisar especificamente os resultados das temperaturas de termômetro de bulbo seco, com variação entre $11,4^{\circ} \mathrm{C}$ a $13,5^{\circ} \mathrm{C}$, os resultados da pesquisa comparados aos critérios (quantitativos e qualitativos) legais e normativos nacionais, em resumo, determinam:

- NR 29: A menor temperatura ambiental para usufruir de pausas para recuperação térmica estabelecidas nessa norma é temperaturas menores a $+10^{\circ} \mathrm{C}$ para uma empresa localizada na zona climática mesotérmica, de acordo com o mapa oficial do IBGE. Ressalta-se que a localização da 
empresa que ocorreu às avaliações de frio enquadra-se na zona climática mesotérmica, ou seja, como não foi mensurado valores do ambiente de trabalho menores de $+10^{\circ} \mathrm{C}$, considerando o critério da NR29, a atividade atende como uma condição que conforme a norma é caracterizada como segura e salubre;

- $\quad$ NR 36: Utiliza o critério do artigo 253 da CLT descrito no próximo item;

- Artigo 253 da CLT: A menor temperatura para usufruir de pausas para recomposição térmica estabelecidas nessa norma é $+10^{\circ} \mathrm{C}$ para uma empresa localizada na zona climática mesotérmica, de acordo com o mapa oficial do IBGE, ou seja, similar ao critério da NR29, a NR 36 e artigo 253 da CLT são atendidos de acordo com os resultados mensurados nesse estudo;

- NR 09: Não especifica parâmetros, porem cita que deve ser avaliado considerando os parâmetros da American Conference of Governmental Industrial Higyenists - ACGIH (2015), que considera que temperaturas acima de $+4{ }^{\circ} \mathrm{C}$ com velocidade do vento calmo, ou seja, abaixo de 2,24 $\mathrm{m} / \mathrm{s}$ os riscos a saúde são baixos. Considerando que os resultados das temperaturas em toda jornada de trabalho foram superior ao critério da NR 09 e que a velocidade do vento foi em média 0,36 m/s, o critério da norma foi atendido considerando que os riscos a saúde dos trabalhadores são baixos.

- NR 15: Método qualitativo por enquadramento do ambiente de trabalho como uma câmara frigorífica ou similaridade a uma câmara frigorifica. De acordo com documentos da empresa (Laudo de Insalubridade da NR 15), o ambiente avaliado não é classificado como uma câmara, pois não se destina a refrigeração de produtos, e sim a manipulação e cortes específicos, local refrigerado, porém não é similar a uma câmara. O critério da norma foi atendido considerando que o ambiente não e insalubre.

Em geral, verifica-se que nenhum dos critérios normativos legais de saúde e segurança no trabalho sugere que seja realizada avaliação de temperatura no corpo do trabalhador.

Verifica-se que considerando as temperaturas mensuradas no ouvido, com média de $36,6^{\circ} \mathrm{C}$, os valores não apresentam evidências de riscos à saúde dos 
trabalhadores, pois de acordo com Simões e Martino (2007) a temperatura ideal situase na faixa entre 36,2 e $37,5^{\circ} \mathrm{C}$. Ainda Simões e Martino (2007) relatam que uma das vantagens de realizar a avaliação de temperatura corporal no tímpano é a proximidade com o hipotálamo e a possibilidade de detectar hipotermia.

Considerando os resultados das variáveis ambientais obtidas nesse estudo, uma taxa de metabolismo igual a $130 \mathrm{~W} / \mathrm{m}^{2}$ e um índice de isolamento térmico (Icl) das vestimentas igual a 0,87 clo, conforme cálculo médio do somatório do índice de resistência térmica das vestimentas fornecidas aos trabalhadores. Esses valores foram obtidos respectivamente na ISO 8996 (2004) para este tipo de atividade e na ISO 9920 (2007) considerando a roupa habitual dos trabalhadores. O resultado médio do $P M V$ ficou $0>P M V<1,0$, o que significa que o trabalhador está em equilíbrio térmico e, teoricamente, em condições de conforto térmico. No entanto, o cálculo de PMV e as avaliações considerando os critérios legais e normativos nacionais, não levam em conta o desconforto causado pela assimetria térmica do meio ambiente ou o contato com superfícies quentes ou frias.

Ao avaliar os resultados obtidos com o sistema de monitoramento de temperatura desenvolvido no LMPT, as avaliações de temperatura nos sítios corporais da mão e pé dos trabalhadores demonstram que comparados aos critérios propostos no estudo de Lehmuskallio, Hassi e Kettunen (2002) que há evidências de riscos à saúde e segurança dos trabalhadores e a condição mais agravante devido à exposição ao frio é representada na saída para o intervalo para refeição, onde os resultados apresentam: Dedos da mão indicando sensação de dor, centro da mão indicando desconforto (resfriado), punho indicando condição neutra, hálux indicando sensação de dor e centro do pé indicando sensação de desconforto (frio).

Também ao verificar os resultados considerando os sítios corporais, as piores condições estão no dedo da mão e no hálux. O dedo da mão apresenta temperatura no início da jornada considerada condição neutra, e em cada momento analisado de interrupção da jornada para usufruir das pausas ou intervalo para refeição, apresentam a condição: frio, dor, frio, dor, frio, dor. O hálux apresenta no início da jornada condição de desconforto (resfriado) e mantém: frio, dor, frio, dor.

Com relação às maiores médias de temperaturas registradas no início da jornada, possivelmente ocorreram devido à falta de estabilização do equipamento e também da estabilização da temperatura do ambiente, pois nos horários que foram 
instalados os equipamentos para medir as temperaturas iniciais da jornada de trabalho ainda não havia entrada de produtos com baixas temperaturas na sala $e$ principalmente contato dos trabalhadores a superfícies frias (mão e pé).

Conforme a pesquisa foi realizada em apenas uma das mãos, pé e ouvido de cada trabalhador, avaliação fundamentada conforme ISO 11079 (2007) "Ergonomics of the termal environment", ressalta-se que conforme estudos atuais realizados com avaliações pontuais de temperatura nas mãos de trabalhadores de frigoríficos, não foram encontrados diferenças significativas de temperatura entre dedos e mãos, que usavam faca ou manipulava produto, e principalmente que não houve correlação estatística significativa entre a temperatura encontrada nos dedos dos trabalhadores com a suas subjetivas percepções térmicas do ambiente de trabalho (RAMOS et al., 2015; ROSÂNGELA; MORO, 2012).

A Figura 01 demonstra o resultado da imagem termográfica, registrada em um horário pontual da jornada de trabalho que foi estabelecido pelos pesquisadores, a Figura representa o resultado da imagem termográfica de um dos participantes do estudo realizado por Ramos et al. (2015).

Figura 1 - Imagens termográficas das mãos de trabalhadores de frigoríficos.

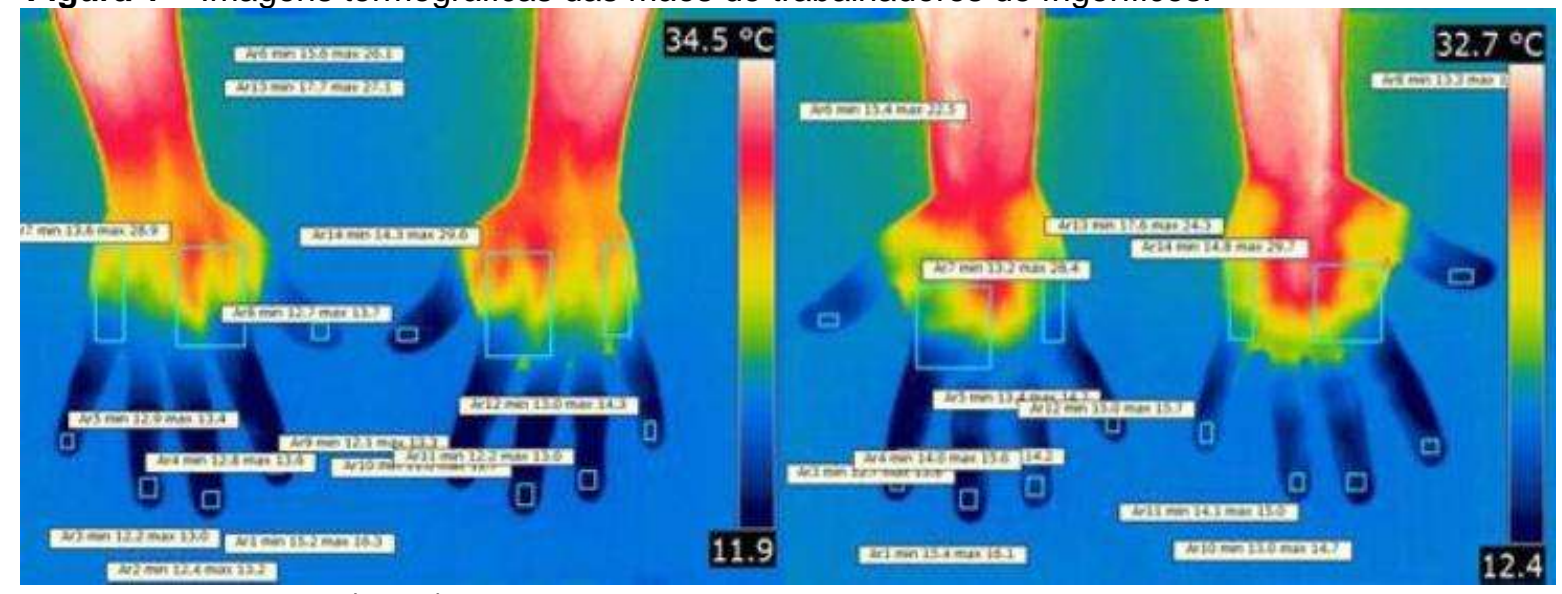

Fonte: Ramos et al. (2015)

Segundo Ramos et al. (2015) é possível verificar na representação das cores que não há diferenças nas cores de temperaturas que possam ser visíveis entre os dedos comparados por cada mão. Importante ressaltar que os resultados foram analisados estatisticamente e comprovaram que não houve diferenças significativas de temperaturas entre os dedos. 
No estudo realizado por Ramos et al. (2015), o tom preto e azul escuro é associado às menores temperaturas (próxima a $12^{\circ} \mathrm{C}$ ) e 0 tom branco e vermelho referente à maior temperatura (próxima a $34,5^{\circ} \mathrm{C}$ ). Temperaturas próximas aos valores encontrados nessa pesquisa.

Os resultados encontrados nessa pesquisa e na pesquisa de Ramos et al. (2015) reforçam que a atividade expõe o trabalhador a riscos devido a exposição ao frio, pois as temperaturas encontradas em ambos os estudos foram similares. Também verifica-se nos resultados que ambos os sistemas de mensuração da temperatura corporal apresentam resultados quantitativos que subsidiam dados relevantes para análise de conforto térmico localizado.

Considerando as pausas para o trabalho em frigoríficos, estabelecida na NR 36 (2016), especificamente no item 36.13.2, para os trabalhadores que desenvolvem atividades diretamente no processo produtivo, ou seja, desde a recepção até a expedição, onde são exigidas repetitividade e/ou sobrecarga muscular estática ou dinâmica do pescoço, ombros, dorso e membros superiores e inferiores, devem ser asseguradas pausas psicofisiológicas conforme tempo diário da jornada. Que conforme jornada de trabalho do frigorifico avaliado caracteriza-se 60 minutos de pausa que foram distribuídas em três pausas de 20 minutos cada.

Embora o objetivo das pausas seja caracterizado como psicofisiológica, percebe-se com os resultados do estudo, nas condições avaliadas, que não há evidências de contribuição para recuperação térmica dos sítios corporais expostos ao frio. Ao avaliar a saída e retorno para usufruir as três pausas, comparando os resultados com os critérios propostos no estudo de Lehmuskallio, Hassi e Kettunen (2002), percebe-se que a única avaliação que houve alteração de uma condição para outra foi a que apresentou uma variação de sensação de dor para sensação de frio nas avaliações do dedo indicador na terceira pausa. Verifica-se também nos resultados que ocorre variações de temperaturas durante os intervalos de pausa, porem não ocorre de forma que seja significante em que a condição passe de sensação de dor para uma condição de conforto. A melhor condição representada nos resultados, porem não em todos os sítios corporais avaliados, pode ser verificada apenas nas variações de resultados entre saída e retorno de intervalo para refeição com tempo total de 60 minutos fora do ambiente frio, no dedo da mão e no hálux não houve alterações significativas, em ambos a condição passou de dor para frio. 
Nas condições avaliadas, com os resultados encontrados, ao comparar aos critérios normativos nacionais (NR 29, NR 36 e artigo 253 da CLT) de pausa térmica, percebe-se que para o modelo de atividade e equipamentos utilizados os critérios normativos nacionais não representam medidas eficientes de redução de desconforto causado devido à exposição ao frio ocupacional.

\section{CONSIDERAÇÕES FINAIS}

O sistema proposto cumpriu os requisitos de utilização, podendo adquirir a temperatura superficial da pele nos sítios corporais escolhidos e as temperaturas e variáveis ambientais necessárias para análise de desconforto térmico. Além disso, o arranjo de termistores e equipamentos não perturbaram a atividade dos trabalhadores participantes dessa pesquisa.

O sistema apresentado foi comprovado como uma maneira fácil e rápida para instalar e confiável para avaliar as condições ambientais e os riscos da exposição ao frio em trabalho com baixas temperaturas.

Os resultados das avaliações realizadas nessa pesquisa mostram que apesar da atividade representar um PMV adequado, atendimento aos critérios legais e normativos nacionais, os resultados da temperatura do dedo da mão e do hálux apresentam condições de frio e dor durante quase toda jornada de trabalho.

Os resultados também demonstram a possibilidade de erro na definição de desconforto e riscos à saúde do trabalhador que um pesquisador ou profissional pode realizar ao avaliar a exposição ao frio considerando os critérios normativos e legais existentes ao avaliar o frio considerando os resultados do ambiente ou uma avaliação de temperatura corporal pontual.

Também é importante destacar que ao avaliar os resultados encontrados nessa pesquisa com as recomendações de pausas térmicas descritas nos critérios normativos nacionais, percebe-se que para os sítios corporais avaliados na mão e pé, nas condições apresentadas de trabalho e de equipamentos utilizados não representam medidas eficientes de conforto térmico. Considerando que as condições ambientais não podem ser alteradas para atender as normativas de higiene alimentar, cabe aos profissionais e empresas realizar a troca dos equipamentos de proteção individual que possuam um índice de resistência térmica adequada às condições de trabalho. 
Por fim, ressalta-se que é importante analisar os dados separadamente considerando os dados exclusos dos outliers, pois esses podem estar vinculados as variáveis de condição fisiológica pessoal, de uso de roupas, condição física, entre outras. Assim como é importante analisar em pesquisas futuras o tempo de exposição que uma sensação passa de um critério para outro como de sensação de frio para sensação de dor, assim como as demais sensações estabelecidas nos critérios de avaliação dessa pesquisa.

\section{REFERÊNCIAS}

ACGIH - American Conference of Industrial Hygienists (ACGIH). Limites de exposição ocupacional (TLVs) para substâncias químicas e agentes físicos \& Índices biológicos de exposição (BEls). Tradução: Associação Brasileira de Higienistas Ocupacionais (ABHO), 2015.

CONSOLIDAÇÃO DAS LEIS DO TRABALHO. Decreto Lei 5452/43. Constituição 1988. Constituição da República Federativa do Brasil: Artigo 253, § 1 Brasília(DF): Senado;

ASHRAE 55. Thermal environmental conditions for human occupancy. American Society of Heating, Refrigerating and Air Conditioning Engineers Inc., 1791 Tullie Circle, NE, Atlanta, GA 30329, 2013.

BRAZILIAN POULTY ASSOCIATION. Poultry Industry.

2015.https://www.brazilianchicken.com.br (Acessado em: 25/Out/2017).

FANGER, Povl Ole. Thermal comfort: analysis and applications in environmental engineering. New York: McGraw-Hill Book Company; 1970.

GÜTHS, Saulo et al. Body Temperature Monitoring System for Slaughterhouse Workers. Advances In Intelligent Systems And Computing, [s.I.], p.96-105, 11 jun. 2017. Springer International Publishing. http://dx.doi.org/10.1007/978-3-319-60011-6 10

HARMSE, Johannes; ENGELBRECHT, Jacobus; BEKKER, Johan. The impact of physical and ergonomic hazards on poultry abattoir processing workers: a review. International Journal Of Environmental Research And Public Health, [s.I.], v. 13, n. 12, p.197-221, 6 fev. 2016. MDPI AG. http://dx.doi.org/10.3390/ijerph13020197

HOLMÉR, Ingvar. Evaluation of thermal stress in cold regions: a strain assessment strategy. In: International symposium on problems with cold work, 1, 1997, Solna. Proceedings. Solna: National Institute for Working Life, 1997.

INSTITUTO BRASILEIRO DE GEOGRAFIA E ESTATÍSTICA (IBGE). Base de dados agregados (SIDRA) Pesquisa Trimestral do Abate de Animais (2016). Disponível em: http://www.sidra.ibge.gov.br/bda/tabela/listabl.asp?c=1093\&z=t\&o=24. (Acesso em: 16 out. 2017). 
INTERNATIONAL ORGANIZATION FOR STANDARDIZATION. ISO 7730. Ergonomics of the Thermal Environment - Analytical Determination and Interpretation of Thermal Comfort Using Calculation of the PMV and PPD Indices and Local Thermal Comfort. Geneva Switzerland, 2005.

INTERNATIONAL ORGANIZATION FOR STANDARDIZATION. ISO 8996. Ergonomics of the thermal environment - Determination of metabolic rate. Geneva, Switzerland, 2004.

INTERNATIONAL ORGANIZATION FOR STANDARDIZATION. ISO 11079. Ergonomics of the thermal environment - Determination and interpretation of cold stress when using required clothing insulation (IREQ) and local cooling effects. Geneva: International Standards Organisation, 2007.

INTERNATIONAL ORGANIZATION FOR STANDARDIZATION. ISO 9920. Ergonomics of the thermal environment - Estimation of the therm insulation and water vapour resistance of a clothing ensemble. Geneva, Switzerland, 2007.

INTERNATIONAL ORGANIZATION FOR STANDARDIZATION. ISO 13732-3. Ergonomics of the thermal environment - Methods for the assessment of human responses to contact with surfaces - Part 3: Cold surfaces. Geneva: International Standards Organisation; 2005.

LEHMUSKALLIO, Eero; HASSI, Juhani; KETTUNEN, Päivi. The skin in the cold. International Journal Of Circumpolar Health, [s.I.], v. 61, n. 3, p.277-286, set. 2002. Informa UK Limited. http://dx.doi.org/10.3402/ijch.v61i3.17475.

MINISTÉRIO DO TRABALHO E EMPREGO. NR 09: Programa de Prevenção de Riscos Ambientais. 77 ed. São Paulo: Atlas, 2016.

MINISTÉRIO DO TRABALHO E EMPREGO. NR 29: Segurança e Saúde no Trabalho Portuário. 77 ed. São Paulo: Atlas, 2016.

MINISTÉRIO DO TRABALHO E EMPREGO. NR 36: Segurança e Saúde no Trabalho em Empresas de Abate e Processamento de Carnes e Derivados. 77 ed. São Paulo: Atlas, 2016.

OLIVEIRA, Paulo Antonio Barros; MENDES, Jussara Maria Rosa. Processo de trabalho e condições de trabalho em frigoríficos de aves: relato de uma experiência de vigilância em saúde do trabalhador. Ciência \& Saúde Coletiva, [s.I.], v. 19, n. 12, p.4627-4635, dez. 2014. FapUNIFESP (SciELO). http://dx.doi.org/10.1590/1413-812320141912.12792014.

PIEDRAHITA, Hugo. Perception of musculoskeletal symptoms in cold-exposed and non-cold exposed workers. Department of Human Work Sciences, Division of Industrial Ergonomics, p. 1-56, 2003.

Portaria no 210 de 10/11/1998. Dispõe sobre o regulamento técnico da inspeção tecnológica e higiênico-sanitária de carnes de aves. Diário Oficial da União, Brasília, 26 nov. 1998.

RAMOS, Eliane et al. Thermographic Analysis of the Hands of Poultry Slaughterhouse Workers Exposed to Artificially Cold Environment. Procedia Manufacturing, [s.I.], v. 3, p.4252-4259, 2015. Elsevier BV. http://dx.doi.org/10.1016/j.promfg.2015.07.408. 
ROSÂNGELA, Buzanello Márcia; PEREIRA, Moro Antônio Renato. Slaughterhouse workers exposed to cold: proposal of reference thermography values for hands. Work, [s.I.], v. 41, n. 1, p.2876-2881, 2012. IOS Press. http://dx.doi.org/10.3233/WOR-2012-0537-2876.

ROSSI, Elisabeth Zumira. Reabilitação e reinserção no trabalho de bancários portadores de LER/DORT : análise psicodinâmica. 2008. 270 f. Tese (Doutorado) - Curso de Programa de Pós Graduação em Psicologia Social do Trabalho e das Organizações, Psicologia, Universidade de Brasília, Brasilia, 2008. Disponível em:

<http://repositorio.unb.br/handle/10482/1340>. Acesso em: 20 nov. 2017.

SALIM, Celso Amorim. Doenças do trabalho: exclusão, segregação e relações de gênero. São Paulo em Perspectiva, [s.I.], v. 17, n. 1, p.11-24, mar. 2003. FapUNIFESP (SciELO). http://dx.doi.org/10.1590/s0102-88392003000100003.

SIMÕES, Ana Leda Bertoncini; MARTINO, Milva Maria Figueiredo de. Variabilidade circadiana da temperatura oral, timpânica e axilar em adultos hospitalizados. Revista da Escola de Enfermagem da Usp, [s.I.], v. 41, n. 3, p.485-491, set. 2007. FapUNIFESP (SciELO). http://dx.doi.org/10.1590/s0080-62342007000300020

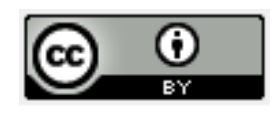

Artigo recebido em: 15/03/2018 e aceito para publicação em: 12/02/2019

DOI: http://dx.doi.org/10.14488/1676-1901.v19i1.3192 